\title{
Canine Oral Osteocartilaginous Malignant Amelanotic Melanoma with Pulmonary Metastasis
}

\author{
Gisele Braziliano de Andrade', Alanderson Rodrigues da Silva ${ }^{2}$, João Bosco Vilela Campos', \\ Joyce Katiuccia Medeiros Ramos Carvalho ${ }^{3}$, Rosalia Marina Infiesta Zulim³ ${ }^{3}$, Luciano Pereira de Barros ${ }^{3}$, \\ Cristiano Marcelo Espinola Carvalho ${ }^{2} \&$ Heitor Miraglia Herrera ${ }^{1,2}$
}

\begin{abstract}
Background: Melanomas are typically malignant neoplasms commonly observed in the oral cavity of dogs. The classical presentation of melanomas with characteristic melanin pigmentation is easy to diagnose; however, in some cases, the lack of melanin production in the amelanotic oral tumors cause a delay in establishing the precocious diagnosis and consequent treatment. The aim of this report was to evaluate the histopathological and immunohistochemical aspects of an oral amelanotic melanoma with osteocartilaginous formation and metastasis in a dog, in a temporal way.

Case: A 10-year-old male German Shepherd dog, presenting mouth bleeding with an amelanotic melanoma located between the upper incisors was received at the Veterinary Hospital of the Catholic University Dom Bosco (UCDB), Campo Grande, MS, Brazil. The animal was clinically evaluated and radiography was performed. The tumor was surgically removed and a sample was collected for histopathological examination that revealed spindloid and some epithelioid morphological cell types surrounded by a fibromatous matrix with moderate amounts of fibrovascular stroma. Approximately 1 month after surgical removal, recovery of the tumor was observed, and a second clinical analysis and collection of sample were performed. These procedures were repeated three times showing the same histopathological characteristics added by myxoid, chondroid, fibromatous tissue, and small groups of chondrocytes as well as central areas of irregular mineralized spicules. X-ray examination revealed proliferative and lytic bone infiltration in the jaw. Immunohistochemical analysis for melanocytic differentiation markers was performed showing positivity to Melan-A, tyrosinase and HMB-45 immunoreactivity, while no S100 reactivity was detected. After 11 months of the first biopsy, pleural effusion and radiopaque disseminated nodules of $1 \mathrm{~cm}$ in the lungs were detected by X-ray. The animal died and necropsy was conducted. Multiple masses were observed in the lung and at the parietal pleura, suggesting lung metastasis by the positivity for Melan-A.

Discussion: The dog was first diagnosed with fibromatous epulis based on the observation of fibroblastic tissue and spindle cells with intense vascularization associated to the site of the tumor and its macroscopic aspect. In the subsequent follow-ups the tumor displayed malignant characteristics observed by recurrence after each surgery, as the tumor returned even larger, aggressive, and infiltrative. From the second biopsy, the histopathological analysis showed the undifferentiated character of epithelioid neoplastic cells, demonstrated by the increase of cartilage and osteoid tissue and the mineral deposit. The phenomenon of the tumor stroma to form cartilage and bone is highlighted here because myxoid change and cartilage formation were frequently observed at the site where amelanotic spindle cells were actively proliferating. It is possible that neoplastic melanocytic cells themselves were involved in the development of the osteocartilaginous areas. Although no cytoplasmic melanin pigmentation was found in the tumor fragments, specific melanocytic markers for melanoma detected neoplastic melanocytes and unmelanized melanosomes. The positive reaction for Melan-A, HMB-45, and tyrosinase in the epithelioid, spindle, and cartilaginous cell groups of the neoplasia indicated amelanotic melanoma with osteocartilaginous differentiation. The negative HMB-45, S100, and tyrosinase expression in lung metastasis may be due to the fact that melanomas express aberrant markers and are also known to display occasional loss of their classic immunophenotype. Amelanotic melanoma can be underdiagnosed due to rapid progression of the tumor allied to the dedifferentiation ability of melanocytes. Thus, the follow up study of cell morphology and immunohistochemical analysis for melanogenic factors can be important determinants in diagnosis.
\end{abstract}

Keywords: dog, immunohistochemistry, oral tumor, X-ray. 
G.B. Andrade, A.R. Silva, J.B.V. Campos, et al. 2018. Canine Oral Osteocartilaginous Malignant Amelanotic Mela-

\section{INTRODUCTION}

Melanomas in the oral cavity are the most malignant neoplasm in dogs worldwide [3,6], in contrary to the occurrence rate $(<2 \%)$ in humans $[8,15]$. The clinical and histopathological aspects of mucosal melanomas in dogs are similar to humans based on the variety of cell morphologies, including epithelioid, spindloid, mixed epithelioid/spindloid or small round blue cell melanocyte [16]. Epithelioid and spindle cell pattern melanomas may be confused with poorly differentiated carcinoma, large cell lymphoma, sarcomas, sarcomatoid carcinomas, or inflammatory scarring process [22]. The range of morphological aspects of amelanotic melanomas requires immunohistochemistry to establish a correct diagnosis [1]. The most useful markers for the identification of melanocytes and melanomas are HMB-45, S100, Melan-A, MITF, and tyrosinase $[8,20]$.

Malignant melanoma with bone or cartilage formation has been widely described in humans for more than 30 years, but only few studies on its occurrence in dogs have been reported $[5,10,14]$. Since then, there have been few or no reports on primary oral melanoma with osteocartilaginous differentiation and metastasis in dogs. The objective of this report was to describe, in a temporal way, the histopathological and immunohistochemical characteristics of an oral amelanotic melanoma in a dog, with osteocartilaginous differentiation and metastasis.

\section{CASE}

A 10-year-old male German Shepherd dog, presenting mouth bleeding was received at the Veterinary Hospital of the Catholic University Dom Bosco (UCDB), Campo Grande, MS, Brazil. At the clinical examination, a small reddish nodule $(1 \mathrm{~cm})$, located in the gums on the incisive upper teeth was observed. The tumor was removed and a sample was collected and fixed in $10 \%$ buffered formalin solution for histopathological analysis. During the subsequent months, clinical examination detected the tumor recurrence and, every two months, four surgeries were performed for tumor removal. A fragment of tumor of each biopsy was sent for histopathological examination. In addition, radiological monitoring was performed to detect the tumor local invasion and lung metastasis. The animal died due to an intense hydrothorax and pulmonary metastasis 11 months after the first surgical intervention.
For radiographs of the incisor and upper canine teeth, an open-mouth rostrocaudal projection was used in ventrodorsal decubitus. The X-ray beam was directed at $30^{\circ}$ for these teeth. For the chest projections, the right-left and ventrodorsal were used.

Fragments of the tumor collected from the five biopsies (times 1 to 5), and from the necropsy (time 6), were fixed in $10 \%$ buffered formalin solution, paraffin-embedded, sectioned at $5 \mu \mathrm{m}$, and sections stained with Hematoxylin and Eosin (H\&E). Specific staining with Alcian blue ( $\mathrm{pH} 2.5)$ for the demonstration of myxoid and cartilaginous material was performed; as well as, Masson's trichrome stain for connective tissue. In order to confirm the diagnosis of amelanotic melanoma, immunohistochemical technique was performed at Time 5 and Time 6 using tissue sections of $3 \mu \mathrm{m}$ thick. Briefly, the sections were placed in the Pre-Treatment (Module PT Link $\left.{ }^{\mathrm{TM}}\right)^{1}$ to process deparaffinization, rehydration and epitope retrieval. Endogenous peroxidase activity was blocked using Peroxidase-Blocking (EnVision ${ }^{\mathrm{TM}}$ FLEX $)^{1}$. Tissue sections were subsequently incubated at room temperature with the HMB- $45^{1}$ monoclonal antibody and tyrosinase ${ }^{1}$ monoclonal antibody to detect early unmelanized melanogenic compartment of melanocytes (melanosomes). The $\mathrm{S} 100^{1}$ polyclonal antibody and Melan- $\mathrm{A}^{1}$ monoclonal antibody were used for the detection of melanocytes and melanocytic neoplastic cells.

Six follow-ups were performed (Time 1 to Time 6) using X-ray, macroscopy, and histopathologic analyses, as detailed below. A common general histological characteristic observed in every biopsy included an invasive pattern, in which the neoplasm extended into the connective tissue and no junctional activity was noted. Malignant cells showed a range of shapes, including spindle and epithelioid-shaped cells with mitotic figures, and no cytoplasmic melanin pigmentation was detected in the tumor fragments.

Time 1. In the first clinical examination, the animal presented a red spongy-like mass of smooth surface with approximately $1 \mathrm{~cm}$ in diameter (Figure 1A). Radiological analysis of the oral cavity demonstrated discrete resorption of the alveolar bone edge incisor, and no change in the lung was detected by chest radiographic examinations. Histopathological analyses showed spindle-cell types surrounded by a fibromatous matrix with moderate amounts of fibrovascular stroma and some neoplastic epithelioid cells (Figure 1B). 
Time 2. At the second follow-up, the tumor increased in size $(3 \mathrm{~cm})$, thus displacing the incisors, and showed sharp edges and necrotic spots on the surface (Figure 2A). There was profuse bleeding at the site of the tumor. Radiological examination revealed an increased space between the front teeth with swelling of soft tissue and loss of normal trabecular pattern next to the incisors, but with no change in the chest. In the histopathological analysis, neoplastic spindle cells were found interspersed by an amorphous material constituted by myxoid, chondroid, and fibromatous tissue; areas of a purple material with epithelioid cells at the periphery were also observed (Figure 2B).

Time 3. At the third follow-up, the tumor increased further in size $(5 \mathrm{~cm})$ with the same gross characteristics presented at Time 2 (Figure 3A). Radiographic examination revealed a marked presence of proliferative and lytic bone on the site, absence of 2 nd and 3rd left incisors, and absence of pulmonary metastasis. The histopathological analysis demonstrated a chondro differentiation of epithelioid neoplastic cells displayed by some cartilaginous light matrix around small groups of round cells (chondrocytes) detected using Alcian blue ( $\mathrm{pH}$ 2.5) staining (Figure 3B). Central areas of a dark blue material indicating irregular mineralized spicules were also observed.

Time 4. At the fourth follow-up, the tumor increased in size $(8 \mathrm{~cm})$ and presented a lobular shape surrounded by some purulent areas (Figure 4A). At this time, a dense connective tissue interspersed by many epithelioid, spindle cells with undelimited cytoplasm and groups of chondrocytes, as evidenced by Masson's trichrome staining, were also noted (Figure 4B).

Time 5. At the fifth follow-up, a full maxillectomy was performed on the animal as the mass relapsed with more intensity (Figure 5A); X-ray examination indicated large bone infiltration in the jaw. After maxillectomy, no lytic reactions or proliferative effects were observed in this bone tissue. Histologically, a predominance of masses of cartilage surrounded by spindle-shaped neoplastic cells (Figure 5B) with marked hyperchromatism, nuclear pleomorphism, and over four mitoses per field were observed. Melan-A positive neoplastic epithelioid and spindle cells were found around and inside the cartilage areas (Figure 5C). We also observed tyrosinase (Figure 5D) and HMB-45 positive immunoreactivity, while no S100 immunoreactivity was detected.
Time 6. The animal presented a strong dyspnea and cyanosis and subsequently died. Pleural effusion and radiopaque disseminated nodules of $1 \mathrm{~cm}$ in the lungs were detected by X-ray examination. However, no tumor was detected in the oral cavity. Radiographic imaging after thoracentesis displayed radiopacity in the left cranial thorax. The necropsy revealed an intense hydrothorax and multiple whitish and firm masses interspersed with reddish and soft areas, ranging from 3 to $10 \mathrm{~cm}$ in diameter, and occupying $80 \%$ of the lung parenchyma (Figure 6A). The microscopic evaluation demonstrated many masses of cartilaginous tissue with few spindle and epithelioid neoplastic cells at the periphery of the parenchyma (Figure 6B). Melan-A positive cells were found around and inside some cartilage cells. In contrast, no positive immunoreactivity to HMB-45, S100, and tyrosinase was observed.

\section{DISCUSSION}

The dog was first diagnosed with fibromatous epulis based on the histopathologic findings of fibroblastic tissue and spindle cells with intense vascularization associated to the site of the tumor and its macroscopic aspect. Indeed, fibromatous epulis is a general clinical term for a gingival growing mass [7].

The recurrence and the malignant characteristics (invasiveness and consequent profuse bleeding) displayed by the tumor in the second follow-up, associated to the histologic aspect of myxoid and chondroid tissue, led us to carry out a more detailed study of the neoplasia. The histopathological analysis of the third biopsy clearly showed the undifferentiated character of epithelioid neoplastic cells, demonstrated by the increase of cartilage and osteoid tissue and the mineral deposit. The myxoid matrix formed by the increasing concentration of mucin, detected here using Alcian blue staining, was considered to be in an early stage of cartilaginous matrix formation [10].

Although no cytoplasmic melanin pigmentation was detected in the tumor fragments, the diagnosis of malignant amelanotic melanoma had to be considered, because its diagnosis is extremely challenging in the absence of melanin pigments [12]. The presence of significant collagen matrix and spindle cells within collagen fibers, well observed in the fourth biopsy, indicated that neoplastic melanocytes actively produced collagen and osteoid backgrounds within the 


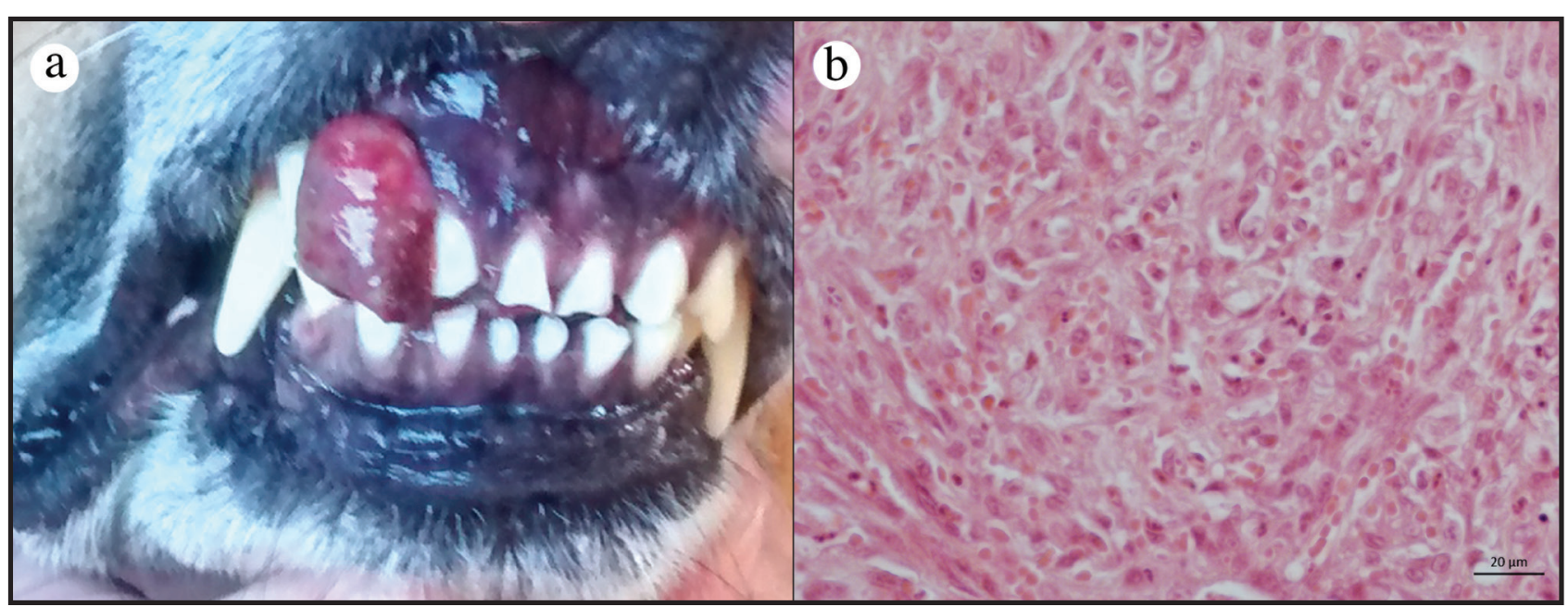

Figure 1. First post-surgery follow-up (Time 1) in a dog with oral amelanotic melanoma. A- Red spongy-like mass on the incisive upper teeth. B- Spindle-cell in a fibromatous matrix and some neoplastic epithelioid cells with a large and clear cytoplasm, and vesicular nuclei with one prominent nucleolus (H\&E).

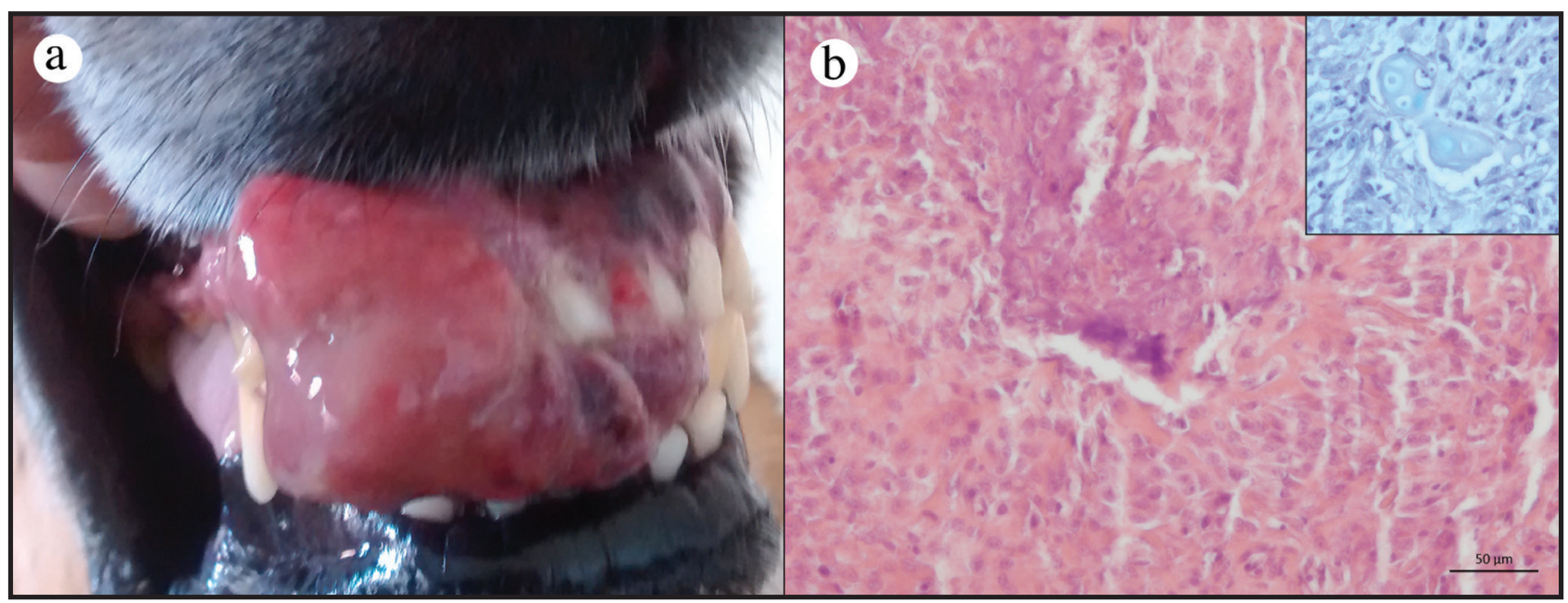

Figure 2. The second follow-up (Time 2) of a dog with oral amelanotic melanoma. A- Sharp edges mass and necrotic spots on the surface. B- Myxoidfibromatous tissue with some spindle and epithelioid cells (H\&E). Inset: Incipient chondrocyte matrix in Alcian Blue.

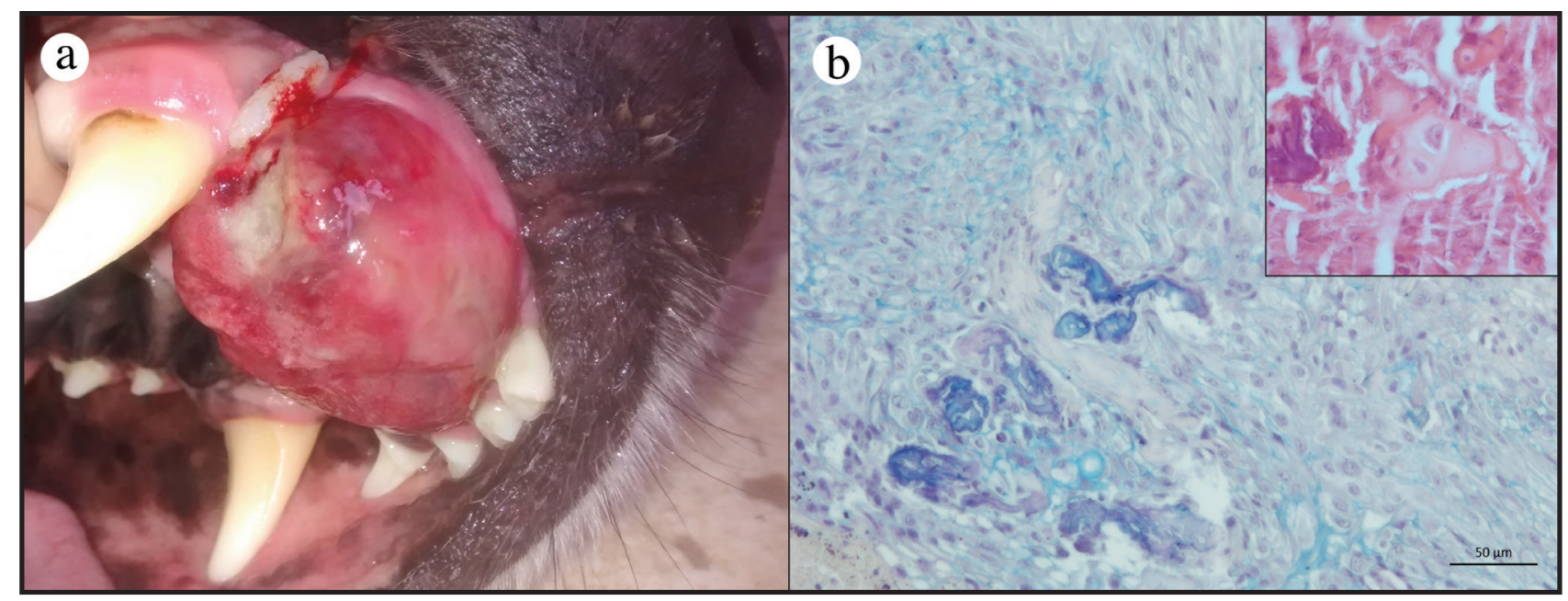

Figure 3. The third follow-up (Time 3) of a dog with oral amelanotic melanoma. A- Increased neoplastic mass with necrotic areas. B- Cartilaginous light matrix and central areas of a dark blue material indicating irregular mineralized spicules in Alcian blue. Inset: Small group of chondrocytes (H\&E). 


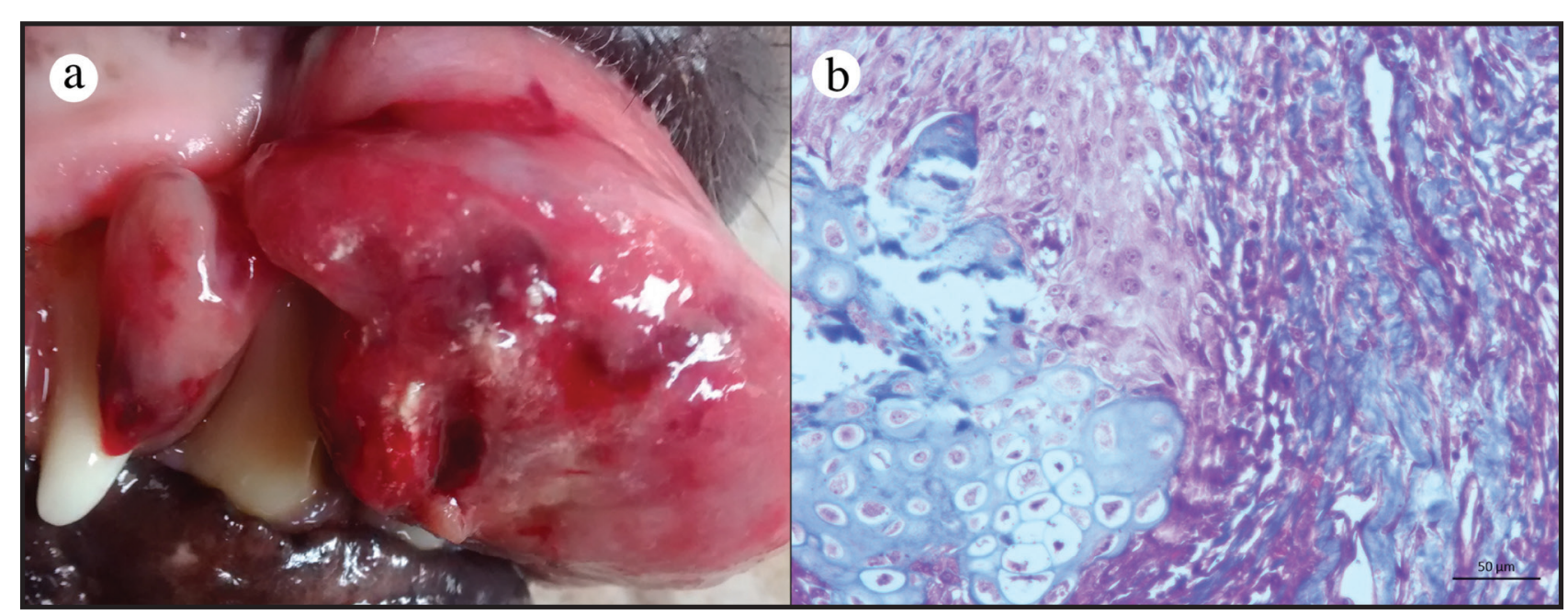

Figure 4. The fourth follow-up (Time 4) of a dog with oral amelanotic melanoma. A- Neoplastic mass presenting lobular shape with purulent areas. B- Dense connective tissue interspersed by epithelioid, spindle cells and groups of chondrocytes in Masson's Trichrome.

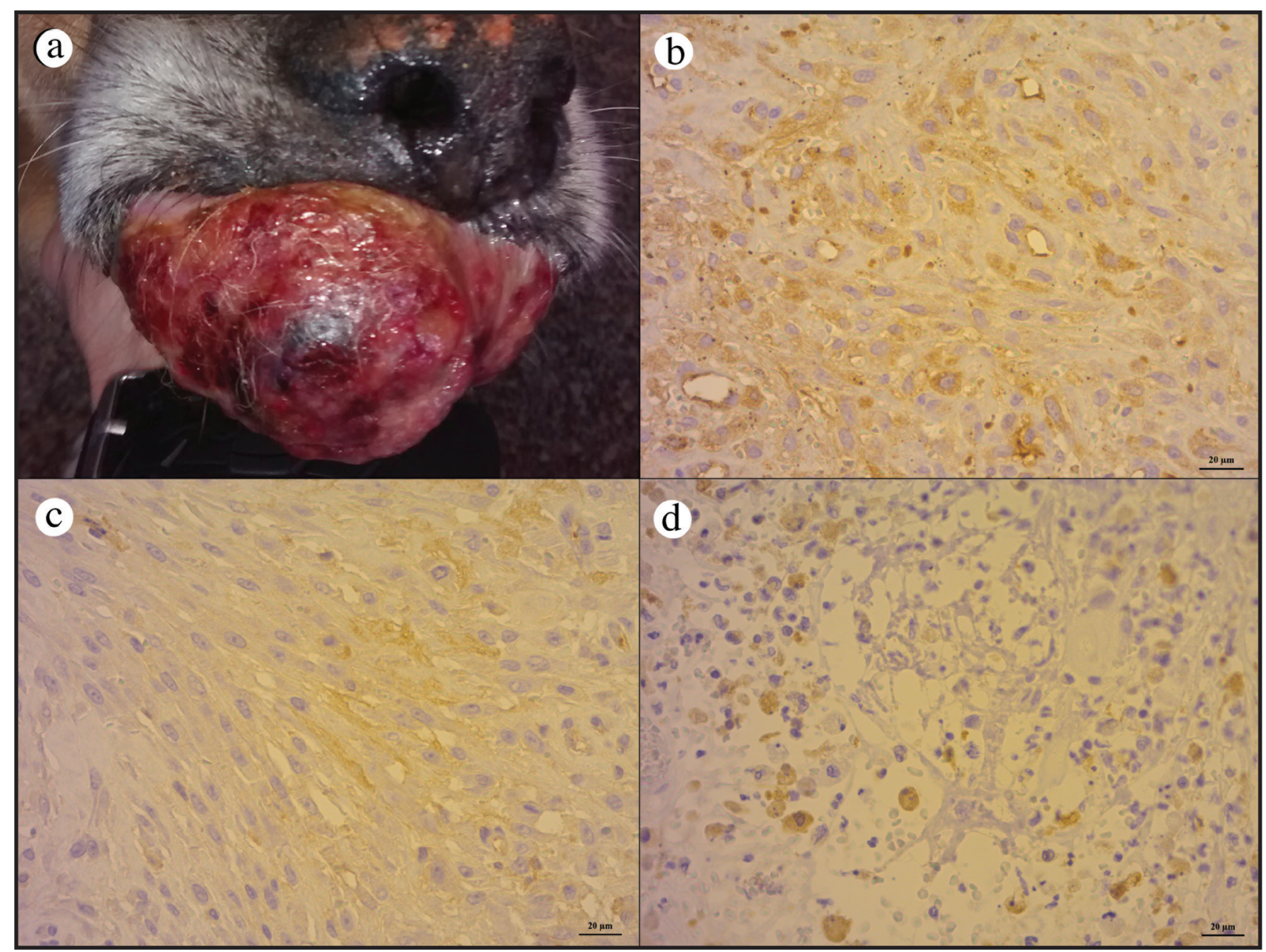

Figure 5. The fourth follow-up (Time 5) of a dog with oral amelanotic melanoma. A- Intense proliferative relapsed tumor mass. B- Granular immunoreactivity to Melan-A was observed. C- Tyrosinase immunoreactivity. D- HMB-45 immunoreactivity.

dense connective tissue. This demonstrated the ability of melanocytes to make mesenchymal elements [5].

It is important to address the fact that after each surgery the tumor returned even larger, aggressive, and infiltrative. As a consequence, a maxillectomy was ne- cessary at Time 5. In this biopsy, the neoplastic cartilage component predominated as demonstrated by irregular masses of cartilaginous matrix, strongly positive for Alcian blue ( $\mathrm{pH} 2.5)$. The phenomenon of the tumor stroma to form cartilage and bone is highlighted here because 


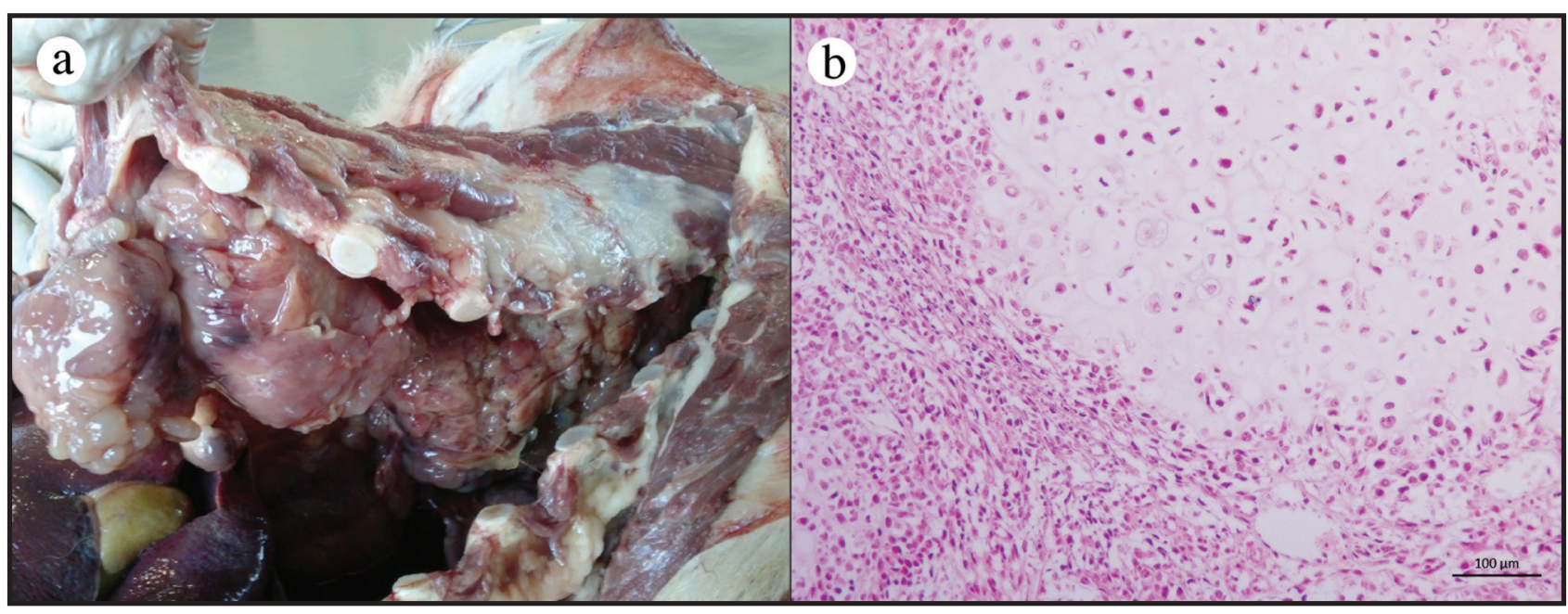

Figure 6. The necropsy (Time 6) examination of a dog with metastatic amelanotic melanoma in thoracic cavity. A- Multiple whitish and firm masses interspersed with reddish and soft areas adhered to parietal pleura occupying $80 \%$ of the lung parenchyma. B- Mass of cartilaginous tissue with few spindle and epithelioid neoplastic cells (H\&E).

myxoid change and cartilage formation were frequently observed at the site where amelanotic spindle cells were actively proliferating [10]. It is possible that neoplastic melanocytic cells themselves were involved in the development of the osteocartilaginous areas. The origin of the osteocartilaginous components is not well established but may result from pseudosarcomatous differentiation of neoplastic melanocytes or from metaplasia of the surrounding stroma in response to osteogenic factors produced during invasion by melanoma cells [14].

Although the immunohistochemical analysis showed a negative workup for $\mathrm{S} 100$ at Time 5, specific melanocytic markers for melanoma such as Melan-A, tyrosinase, and HMB-45 detected the presence of neoplastic melanocytes and unmelanized melanosomes. These markers have been considered useful for dog and human melanoma, and have a high specificity of 95$100 \%[9,19]$. Moreover, the S100 negative melanomas occurred in up to $4 \%$ of the cases because $\mathrm{S} 100$ protein is not specific to melanoma, but also stains peripheral nerve sheath tumors, some sarcomas, salivary gland carcinomas, Langerhans cell histiocytosis, and other hematolymphoid tumors $[4,9]$.

The negative HMB-45, S100, and tyrosinase expression in lung metastasis at Time 6, with preservation of immunoreactivity of the melanocytic marker Melan-A, may be due to the proliferation of a cell subpopulation that was not present in the oral biopsies. Melanomas are well known to express aberrant markers and are also known to display occasional loss of their classic immunophenotype, thus causing problems for histopathological diagnosis [2]. Metastasis in melanoma has a propensity for antigenic shift; therefore, negative or low intensity labeling cases can be interpreted as poorly differentiated and highly malignant tumors [11,13].

The tumor showed a local invasive behavior, confirmed by X-ray, but no metastasis to local lymph nodes was identified, which is usually described to be a primary site for malignant melanomas and other malignancies of the head [18]. There are two points that deserve comments. First, pulmonary changes by $\mathrm{X}$-ray were only detected 1 year after the appearance of the tumor in the oral cavity. Thus, it is necessary to use more accurate techniques of image analysis in order to detect early tumor staging and provide better prognosis [17]. Second, the fact that the tumor was resected four times to improve the animal's feeding could have contributed to the dissemination of neoplastic cells. Indeed, previous studies have reported that biopsies may be unfavorable to the clinical course of the disease [21].

In the present case, the neoplastic pattern was consistent with an osteocartilaginous amelanotic melanoma. The present report is one of only few that describes this disease in dogs. Amelanotic melanoma can be underdiagnosed due to rapid progression of the tumor allied to the dedifferentiation ability of melanocytes. In this regard, the use of immunohistochemical markers was determinant in the diagnosis because, even in the most advanced stage, the neoplasia did not present macroscopic and microscopic features of melanoma.

\section{MANUFACTURER}

${ }^{1}$ Dako. Carpinteria, CA, USA

Declaration of interest. The authors report no conflicts of interest. The authors alone are responsible for the content and the writing of the paper. 


\section{REFERENCES}

1 Agaimy A., Specht K., Stoehr R., Lorey T., Märkl B., Niedobitek G., Straub M., Hager T., Reis A.C., Schilling B., Schneider-Stock R., Hartmann A. \& Mentzel T. 2016. Metastatic malignant melanoma with complete loss of differentiation markers (undifferentiated/dedifferentiated melanoma). The American Journal of Surgical Pathology. 40(2): 181-191.

2 Banerjee S.S. \& Harris M. 2000. Morphological and immunophenotypic variations in malignant melanoma. Histopathology. 36(5): 387-402.

3 Bergman P.J. 2007. Canine oral melanoma. Clinical Techniques in Small Animal Practice. 22(2): 55-60.

4 Biernacka A., Linos K.D., DeLong P.A., Suriawinata A.A., Padmanabhan V. \& Liu X. 2016. A case of S-100 negative melanoma: A diagnostic pitfall in the workup of a poorly differentiated metastatic tumor of unknown origin. Cytojournal. 13(1): 21.

5 Ellis A.E., Harmon B.G., Miller D.L., Northrup N.C., Latimer K.S. \& Uhl E.W. 2010. Gingival osteogenic melanoma in two dogs. Journal of Veterinary Diagnostic Investigation. 22(1): 147-151.

6 Esplin D.G. 2008. Survival of dogs following surgical excision of histologically well-differentiated melanocytic neoplasms of the mucous membranes of the lips and oral cavity. Veterinary Pathology. 45(6): 889-896.

7 Gardner D.G. 1996. Epulides in the dog: a review. Journal of Oral Pathology and Medicine. 25(1): 32-37.

8 Ohnishi Y., Watanabe M., Fujii T., Sunada N., Yoshimoto H., Kubo H., Wato M. \& Kakudo K. 2015. A rare case of amelanotic malignant melanoma in the oral region: Clinical investigation and immunohistochemical study. Oncology Letters. 10(6): 3761-3764.

9 Ordóñez N.G. 2014. Value of melanocytic-associated immunohistochemical markers in the diagnosis of malignant melanoma: a review and update. Human Pathology. 45(2): 191-205.

10 Oyamada T., Tanaka H., Park C.H., Ueki H., Komiya T. \& Arai S. 2007. Pathology of canine oral malignant melanoma with cartilage and/or osteoid formation. Journal of Veterinary Medical Science. 69(11): 1155-1161.

11 Plaza J.A., Suster D. \& Perez-Montiel D. 2007. Expression of immunohistochemical markers in primary and metastatic malignant melanoma: a comparative study in 70 patients using a tissue microarray technique. Applied Immunohistochemistry and Molecular Morphology. 15(4): 421-425.

12 Ramos-Vara J.A., Beissenherz M.E., Miller M.A., Johnson G.C., Pace L.W., Fard A. \& Kottler S.J. 2000. Retrospective study of 338 canine oral melanomas with clinical, histologic, and immunohistochemical review of 129 cases. Veterinary Pathology. 37(6): 597-608.

13 Rolim V.M., Casagrande R.A., Watanabe T.T., Wouters A.T., Wouters F., Sonne L. \& Driemeier D. 2012. Amelanotic melanoma in dogs: a retrospective study of 35 cases (2004-2010) and immunohistochemical characterization. Pesquisa Veterinária Brasileira. 32(4): 340-346.

14 Sanchez J., Ramirez G.A., Buendia A.J., Vilafranca M., Martinez C.M., Altimira J. \& Navarro J.A. 2007. Immunohistochemical characterization and evaluation of prognostic factors in canine oral melanomas with osteocartilaginous differentiation. Veterinary Pathology. 44(5): 676-682.

15 Sedassari B.T., Lascane N.A.S., Freitas A.L.S., Mautoni M.C., Sotto M.N., Gallottini M.H.C., Sousa S.C.O.M. \& Pinto D.S. 2016. In situ melanoma of the gingiva associated with dense inflammation and pigment deposition: A potential diagnostic pitfall in evaluating stromal invasion. Head and Neck Pathology. 10(4): 547-551.

16 Simpson R.M., Bastian B.C., Michael H.T., Webster J.D., Prasad M.L., Conway C.M., Prieto V.M., Gary J.G., Goldschmidt M.H., Esplin D.G., Smedley R.C., Piris A., Meuten D.J., Kiupel M., Lee C.R., Ward J.M., Dwyer J.E., Davis B.J., Anver M.R., Molinolo A.A., Hoover S.B., Rodriguez-Canales J. \& Hewitt S.M. 2014. Sporadic naturally occurring melanoma in dogs as a preclinical model for human melanoma. Pigment Cell and Melanoma Research. 27(1): 37-47.

17 Singh H., Kumar P., Augustine J., Urs A.B. \& Gupta S. 2016. Primary malignant melanoma of oral cavity: A report of three rare cases. Contemporary Clinical Dentistry. 7(1): 87-89.

18 Skinner O.T., Boston S.E. \& Souza C.H.D.M. 2017. Patterns of lymph node metastasis identified following bilateral mandibular and medial retropharyngeal lymphadenectomy in $31 \mathrm{dogs}$ with malignancies of the head. Veterinary and Comparative Oncology. 15(3): 881-889.

19 Smedley R.C., Lamoureux J., Sledge D.G. \& Kiupel M. 2011. Immunohistochemical diagnosis of canine oral amelanotic melanocytic neoplasms. Veterinary Pathology. 48(1): 32-40. 
20 Smith M.H., Bhattacharyya I., Cohen D.M., Islam N.M., Fitzpatrick S.G., Montague L.J., Damm D.D. \& Fowler C.B. 2016. Melanoma of the oral cavity: an analysis of 46 new cases with emphasis on clinical and histopathologic characteristics. Head and Neck Pathology. 10(3): 298-305.

21 Tohme S., Simmons R.L. \& Tsung A. 2017. Surgery for cancer: a trigger for metastases. Cancer Research. 77(7): 1548-1552.

22 Yu C.H., Chen H.H., Liu C.M., Jeng Y.M., Wang J.T., Wang Y.P., Liu B.Y., Sun A. \& Chiang C.P. 2005. HMB-45 may be a more sensitive maker than S-100 or Melan-A for immunohistochemical diagnosis of primary oral and nasal mucosal melanomas. Journal of oral Pathology and Medicine. 34(9): 540-545. 